\title{
Stable Attitudes and Behaviors as Boundary Conditions of Reinforcing Spirals Models
}

\author{
Michael Scharkow ${ }^{1} \&$ Marko Bachl ${ }^{2}$ \\ ${ }^{1}$ Zeppelin University \\ ${ }^{2}$ University of Hohenheim
}

Draft, 2018-05-02

\begin{abstract}
The reinforcing spirals model (RSM) has quickly become a popular way of thinking about the reciprocal dynamics of media use and effects, not only in political communication. One key assumption of the model is that both exposure to media content and individual attitudes vary over time - if there is no variation, there cannot be any covariation. In this paper, we discuss the assumptions of the RSM regarding intra-individual variation and inter-individual differences in media use and political attitudes. We present empirical results on the temporal stability of different phenomena of interest in political communication, such as media use, campaign involvement, or candidate evaluations, drawing on panel data from two national election studies in the USA and Germany. Our results suggest that, after accounting for imperfect measurement, media use and most political orientations and attitudes are very stable over the course of a campaign, making it rather unlikely to detect patterns corresponding to the RSM. We discuss the usefulness of the RSM and similar dynamic media effects models in light of our results.
\end{abstract}

Keywords: Reinforcing Spirals Model, Stability, Reliability, GLES, ANES

Prepared for the conference proceedings of the 2017 annual meeting of the DGPuK Methods section, "Dynamische Prozesse der öffentlichen Kommunikation: Methodische Herausforderungen", edited by Stefan Geiß, Christian Schemer, Teresa K. Naab, Christina Peter, and Philipp Müller.

Correspondence concerning this article should be addressed to Michael Scharkow, Department of Culture and Communication, Zeppelin University, Friedrichshafen, Germany. E-mail: michael.scharkow@zu.de 
Many theories and models of media effects describe dynamic processes in which media selection, media exposure, and attitudes mutually influence one another over time. The reinforcing spirals model (RSM, Slater, 2007, 2015, 2017) has become a popular way of thinking about such reciprocal dynamics of media use and effects: Higher exposure to certain media messages leads to attitude change, which in turn leads to selective media use and higher exposure to similar messages, which in turn reinforces the attitude change, and so on. Moeller and de Vreese (2015), for example, investigated whether learning from the news and political news use reinforce each other over time in a sample of Dutch adolescents. The growth processes of political knowledge and news use were found to be interrelated, indicating a "spiral of political learning" (p. 1). Schemer (2012) used a similar approach to model the longitudinal relationship between negative affects toward asylum seekers and attention to political advertisement in a Swiss referendum campaign.

One key assumption in any RSM is that both exposure to media content and individual attitudes vary over time - if there is no variation, there cannot be any covariation. However, the relative stability of media use, attitudes, and behaviors is often ignored in RSM studies. Taking political communication as a prominent example, we present empirical evidence that news media use and many relevant self-report measures were exceedingly stable over the course of typical panel studies during two election campaigns in the USA and Germany. The rank-order of individuals hardly changed and the individual growth processes showed almost no variation. This makes it rather unlikely to find any meaningful covariation of the variables over time, let alone evidence for the patterns, which are predicted by the RSM. We conclude with theoretical as well as methodological reflections on whether and how to refine and empirically test the RSM and similar dynamic media effects models in light of our results.

\section{Preconditions of (Statistical) Reinforcing Spirals Models}

Empirical tests of individual-level dynamic models such as the RSM of course require longitudinal designs with repeated measures of the same constructs from the same individuals. A minimum of three consecutive measurements per person is necessary to establish the complete process as proposed in Slater's $(2007,2015,2017)$ conceptual work. The data is most commonly analyzed with cross-lagged panel models (CLPM; see, e.g., Figure 1 in Slater, 2007) or variants of parallel latent growth-curve models (PLGCM; e.g., Moeller \& de Vreese, 2015; Schemer, 2012; Slater \& Hayes, 2010). Specific patterns of (temporal) covariation of media use and relevant attitudes or behaviors (usually multiple cross-lagged paths in CLPM and covariation of slopes in PLGCM) are taken as evidence in favor of reinforcing spirals. A necessary pre-condition for detecting any kind of covariation-both contemporary or over time - is, of course, that the measures or derived latent variables themselves vary at all. This concerns both CLPM and PLGCM and is especially important if we are interested in causal processes after accounting for previous measures of the same construct (i.e., its stability). If the measures of interest are highly stable, then there is just no way to empirically distinguish between homeostasis (caused by negative feedback loops which occurred in between the waves) and causal independence (no reinforcing process took place). We therefore aim to answer two basic research questions to clarify the importance of stable attitudes and behaviors as boundary conditions in RSM research: 
1. How stable are typical election-study measures when controlling for reliability?

2. How variable are within-person growth trajectories of typical election-study measures?

We take political communication as a suitable example, because the RSM is frequently applied in this domain and because high-quality data sets are publicly available.

\section{Methods}

\section{Data and measures}

We conducted a secondary analysis of two established panel studies: the 2013 Short-term Campaign Panel from the German Longitudinal Election Study (GLES13; Rattinger et al., 2016) and the 2008-2009 Panel Study from the American National Election Study (ANES08; The American National Election Studies, 2010). The data from these studies are not only of very high quality, but also structurally very similar to many published studies that implicitly or explicitly refer to the reinforcing spirals model (Moeller \& de Vreese, 2015; Schemer, 2012). In line with these studies, we selected $k=33$ measures to test, including media use variables (television, newspaper, Internet use), measures related to attitudes toward parties and candidates, and political self-assessments such as interest in politics. In order to estimate the temporal stability and variability of these measures, at least three repeated measurements are required. This led to the exclusion of some measures that were only measured once or twice during the course of the study. Where available, we selected three equally spaced pre-election waves for each measure, although in some cases unequal time lags were unavoidable. The procedure yielded three-wave panel data with measurement occasions roughly every two months for most of the variables. The average sample sizes across all measures were $n=3,521$ (GLES13) and $n=1,257$ (ANES08). Table 1 summarizes the selected measures. Details about design, sampling, and measurements are available from the documentations of the primary researchers, and our transformations and statistical analyses are available in the replication material. ${ }^{1}$

\section{Analysis}

For every variable in the two data sets, we estimated four different measures of temporal stability and reliability. In general, one can distinguish between test-retest reliability, rank-order (or covariance) stability and mean stability over time. Test-retest reliability is commonly defined as the correlation $r_{12}$ between two repeated measures. However, using this simple correlation as a reliability measure assumes perfect stability, i.e. no true change between measurement occasions. This assumption is not only unfounded in general, but contradicts the theoretical reasoning of any RSM study. Heise (1969) developed a model that avoids this assumption and can separate reliability from stability. The Heise model requires three repeated measures to be identified. Using only the correlations between the measurements, one reliability estimate and two stability estimates can be computed using the following formulas (see Alwin (2007) for a detailed discussion).

$$
S t a b_{12}=\frac{r_{13}}{r_{23}} \quad \text { and } \quad S t a b_{23}=\frac{r_{13}}{r_{12}} ; \quad R e l=\frac{r_{12} * r_{23}}{r_{13}},
$$

${ }^{1}$ https://osf.io/rzeg6/ 
Table 1

Measures used in the secondary analysis

\begin{tabular}{lll}
\hline Variable & ANES08 & GLES13 \\
\hline Media Use & 3 & 3 \\
Evaluation Parties & 2 & 2 \\
Opinion Polarization Parties & 1 & 1 \\
Evaluation Top Candidates & 2 & 2 \\
Opinion Polarization Top Candidates & 1 & 1 \\
Evaluation Government & 1 & 1 \\
Left-Right Classification Top Candidates & 2 & 2 \\
Perceived Polarization Top Candidates & 1 & 1 \\
Interest In Politics & 1 & 1 \\
Interest In The Election Campaign & NA & 1 \\
Conversations About Politics & 1 & 1 \\
Left-Right Self-Classification & 1 & 1 \\
\hline
\end{tabular}

Note. Cells show the number of distinct variables selected.

where $r$ is a correlation and the subscripts denote waves in a three-wave panel survey. We present the results for the stability between waves 1 and 2 in this report and note that the results for $S_{t a b}$ are substantially identical. The Heise (1969) model can also be specified as a path model, specifically an autoregressive model where prior values predict the subsequent measurements. This autoregressive or simplex model is the major component of the cross-lagged panel model (CLPM) that has historically been used to model reciprocal relationships between two measures over time. It is often assumed that the cross-lagged paths from $X_{1}$ to $Y_{2}$ and vice versa are indicative of mutual reinforcement. This, however, requires that not all variance at wave 2 is explained by the autoregressive path, i.e. that both $X$ and $Y$ are not perfectly stable. For example, when $S t a b_{Y}=.90$, even under perfect circumstances $X$ can at most explain the 20 percent remaining variance of $Y$. This is true for all regression models that use lagged dependent variables, and therefore also for the CLPM.

In classical test theory, the observed score $X$ is the sum of the true score $\tau_{X}$ and random error $e_{X}$, and the correlation between consecutive measures is influenced by both the stability of the true scores and the amount of error variance. A measure with low reliability might show high variance (i.e. low stability) over time, but still cannot be explained by a causal process, because the variation over time is mainly due to random noise. In other words, without accounting for measurement error, researchers will underestimate the stability of a measure $Y$ and falsely conclude that there are many rank-order changes that could be explained by $X$. Therefore, it is necessary to separate stability and reliability, for example using the Heise (1969) model, and look at how they might influence the results of a CLPM.

Until now, we were only concerned with covariance-based models and rank-order stability. These do not consider changes in means at all, i.e. perfect rank-order stability is consistent not only with mean stability, but also with homogeneous growth or decline. Since the CLPM does not even include parameters for means, this model cannot say anything 
about whether, for example, interest in the campaign increases or declines over time. In terms of the RSM, it is impossible to distinguish whether there is homeostasis or a spiralingout-of-control process over time using the CLPM. Therefore, a second model class, the latent growth curve model, has been employed to identify reinforcing spiral processes. Just like the CLPM is composed of two simplex models, the PLGCM is composed of two simple growth curve models for $X$ and $Y$.

The simple growth curve model can be defined as a multilevel regression with a random effects and a time predictor (Grimm, Ram, \& Estabrook, 2016),

$$
y_{t, i}=\beta_{00}+\beta_{10} * \text { time }_{t, i}+u_{0 i}+u_{1 i} * \text { time }_{t, i}+e_{t, i},
$$

where the $t$ subscript denotes the measurement occasion (in our model scaled in months) and the $i$ subscript denotes the individual respondent. Since the selected measures had different response scales, we transformed them before estimating the latent growth curve model. The variables were re-scaled by units of standard deviations, so that the effect sizes for the time predictor can roughly be understood as Cohen's $d$ growth per month (Feingold, 2009). Although absolute growth or decline of the selected measures over time might be of interest for applied research, we are mainly interested in the $u_{1 i}$ term, which quantifies the between-person variation in within-person growth. A relationship between the growth curves in a PLGCM can only be established if the individuals differ in their trajectories. In other words, if there is no variation in growth curves, there is no covariation to observe and explain.

In total, we estimated one simplex model and one latent growth curve model for every selected measure, which yielded four estimates plus their standard errors for our analysis: (1) rank-order stability, (2) reliability, (3) linear growth, and (4) variance in linear growth. We used $R$ (R Core Team, 2016), the lme4 (Bates, Maechler, Bolker, \& Walker, 2017) and lavaan (Rosseel, 2017) packages for estimation, and ggplot2 (Wickham, 2009) for graphical summaries.

\section{Results}

In a first step, we look at the rank-order stability and test-retest reliability estimates from the Heise (1969) model. The rank order of respondents in both studies was exceedingly stable for most of the attitudes and behaviors we analyzed. The average rank-order stability estimates for the ANES 2008 were $M=0.91(S D=0.05)$, for the GLES 2013 the stability was even higher with $M=0.94(S D=0.03)$. There were few meaningful differences for media use, political attitudes, and political self-assessments (see Figure 1). Only the assessments of the political positions of the candidates were less stable. This could reflect actual changes in candidates' positions or at least noticeable swings in their perception during the course of a campaign. Overall, a researcher trying to model reinforcing spirals of political attitudes and media exposure with a CLPM would find relatively little variance (about 20 percent on average) left to explain after accounting for rank-order stability and measurement error.

Looking at the reliability coefficients from the Heise model in Figure 2, we obtained strongly varying test-retest reliability estimates for both the ANES 2008 ( $M=0.79$, $S D=0.10)$, and the GLES $2013(M=0.82, S D=0.09)$. Most media use measures and selfassessments were of sufficient reliability. In contrast, the questions about candidate positions 


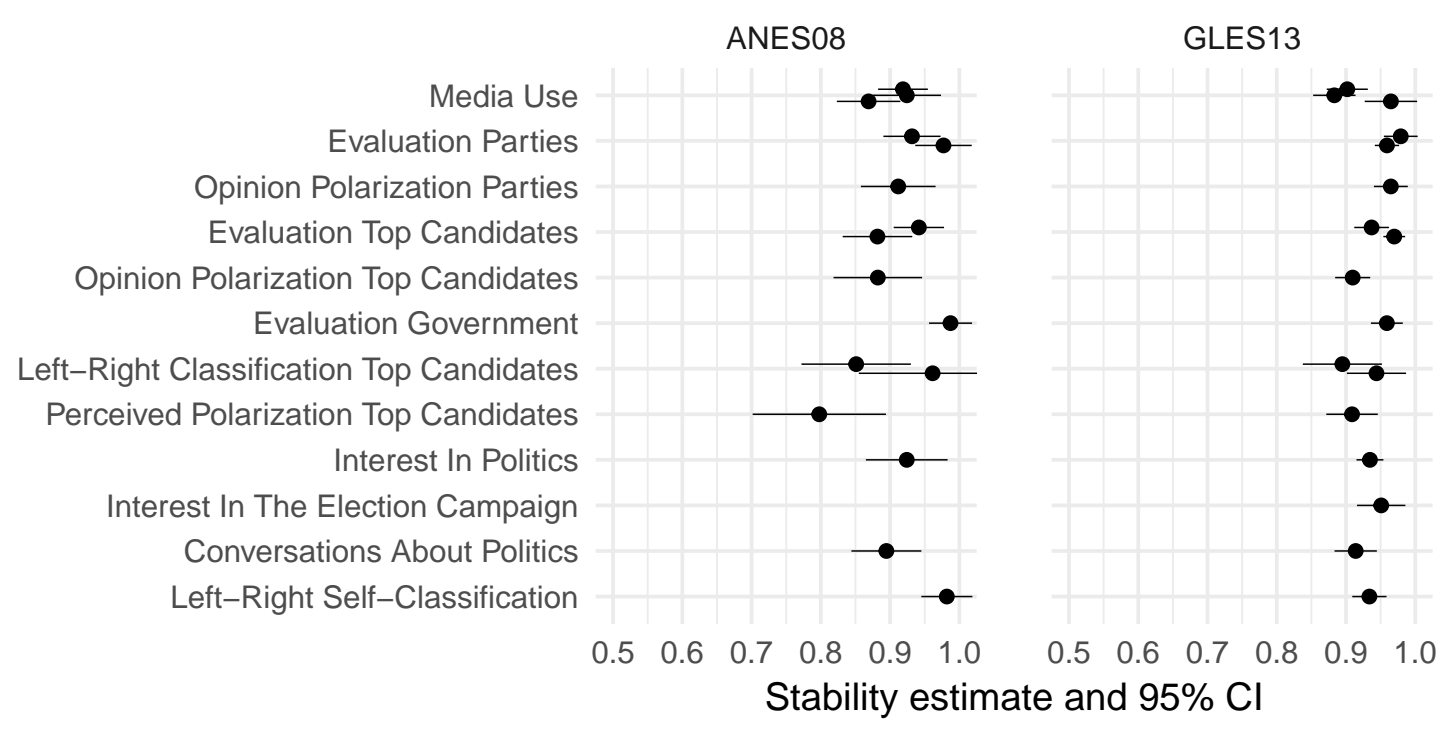

Figure 1. Rank-order stability estimates

and political polarization showed greater shares of measurement error. The combination of high stability and imperfect reliability poses serious problems for an adequate application of the CLPM. If one fails to account for measurement error, the magnitude of the autoregressive path will be underestimated, which incorrectly implies that there is sufficient variation left to explain with cross-lagged paths from other predictors. However, such models would mainly try to fit measurement noise instead of true effects, quite likely leading to either null findings or false positive results.

Given the high stability and moderate-to-high reliability estimates from the Heise model, there seems to be little reason to expect notable media or selection effects in CLP models of political attitudes. Following Schemer, Matthes, and Wirth (2009), it can be useful to look at individual growth trajectories in the context of media effects research. However, our results show that no only the overall rank-order was very stable, there was also little indication of mean variation over time. Figure 3 shows descriptively that the overall levels of almost all variables remained largely unchanged over the course of the campaigns. Even average campaign interest or perceived polarization remain stable over time, therefore homogeneous growth or decline as cause for high rank-order stability can be ruled out. By and large, media use and political attitudes in both campaign studies were rather constant over time.

This finding is supported by the near-zero population-level point estimates of the growth curves' slopes (Figure 4). The overall average slope was 0 . The largest estimated linear growth was $d=0.08$ per month, the strongest decrease was $d=-0.10$, both in the GLES 2013 study. However, there were no systematic differences between the different types of variables. Moreover, there was very little variation in these slopes, as indicated by the width of the intervals for each measure. The aggregate stability was mostly not the result of changes in opposing directions which cancel each other out, but of rather homogeneously flat individual trajectories. The lack of variation was stronger in the ANES 2008 study than 


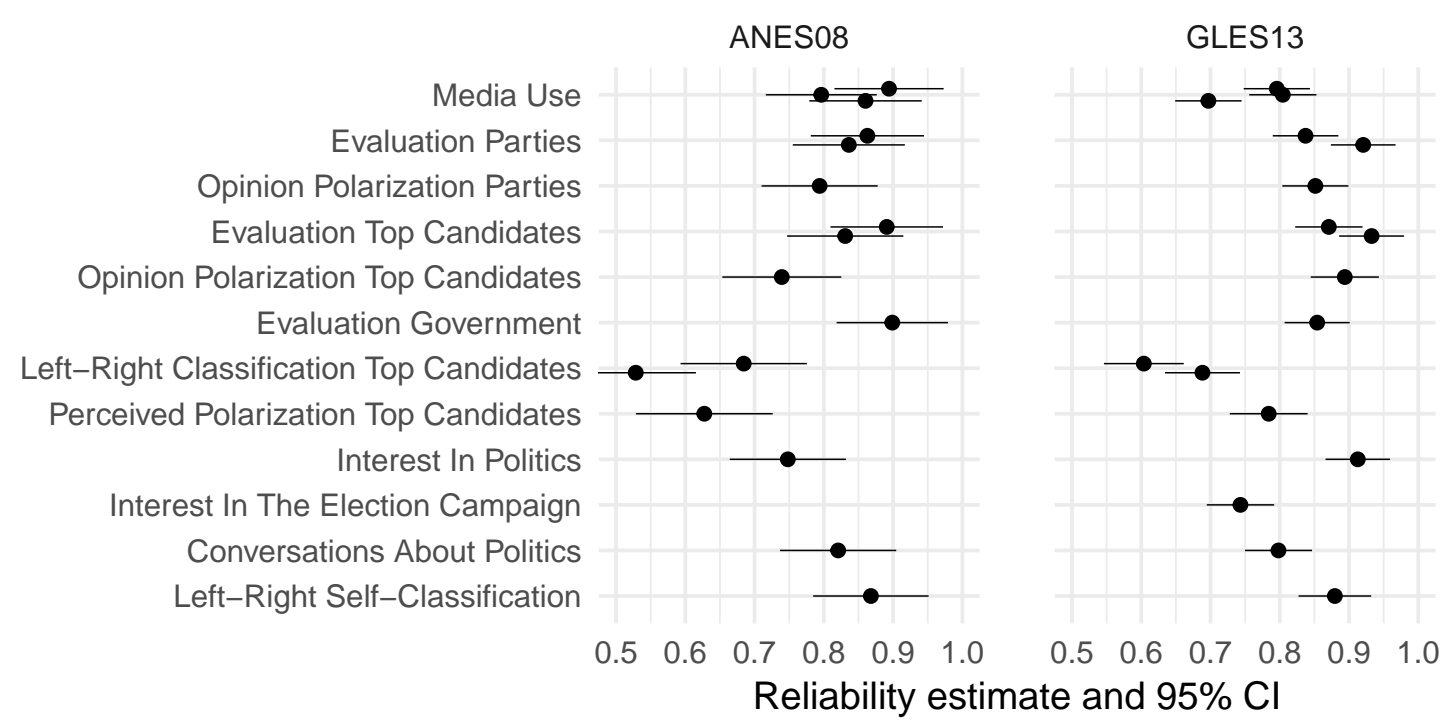

Figure 2. Test-retest reliability estimates
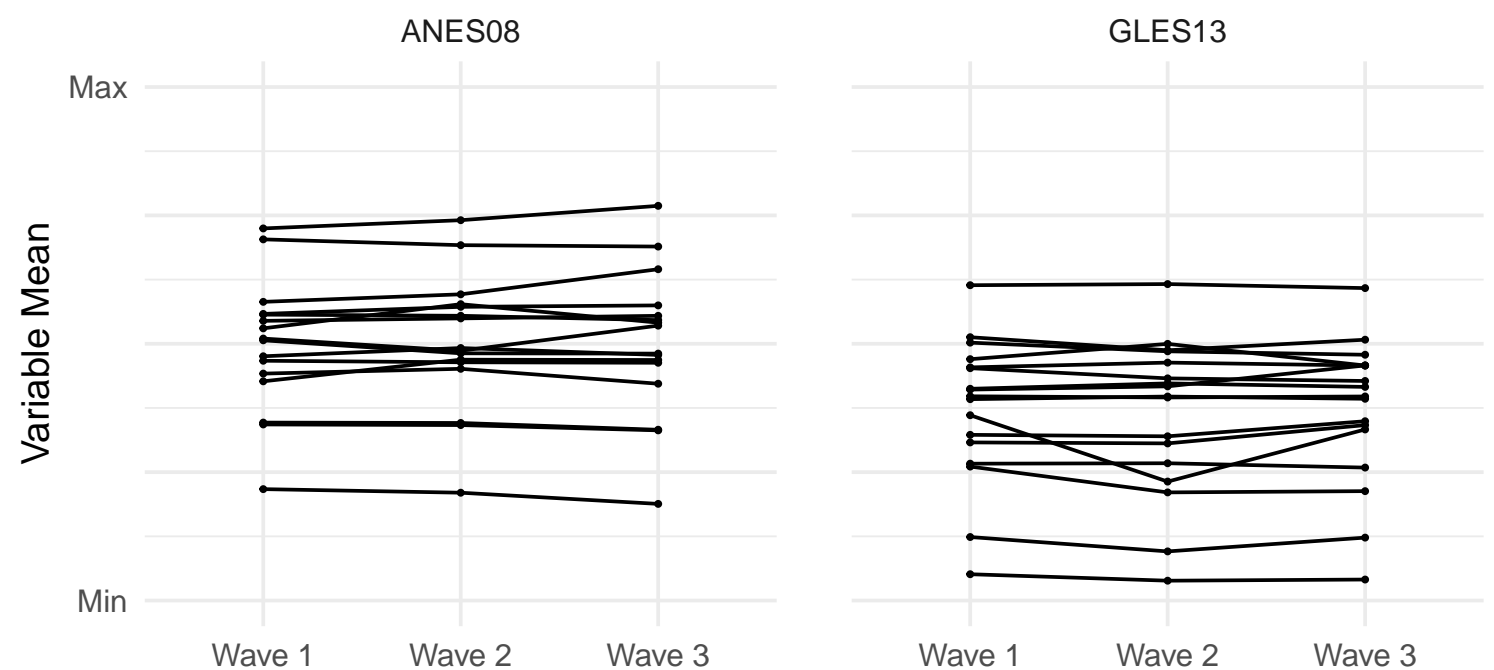

Figure 3. Mean stability

Notes. All variables were rescaled for the plot so that 0 is the empirical minimum and 1 is the empirical maximum of each variable across all waves. 


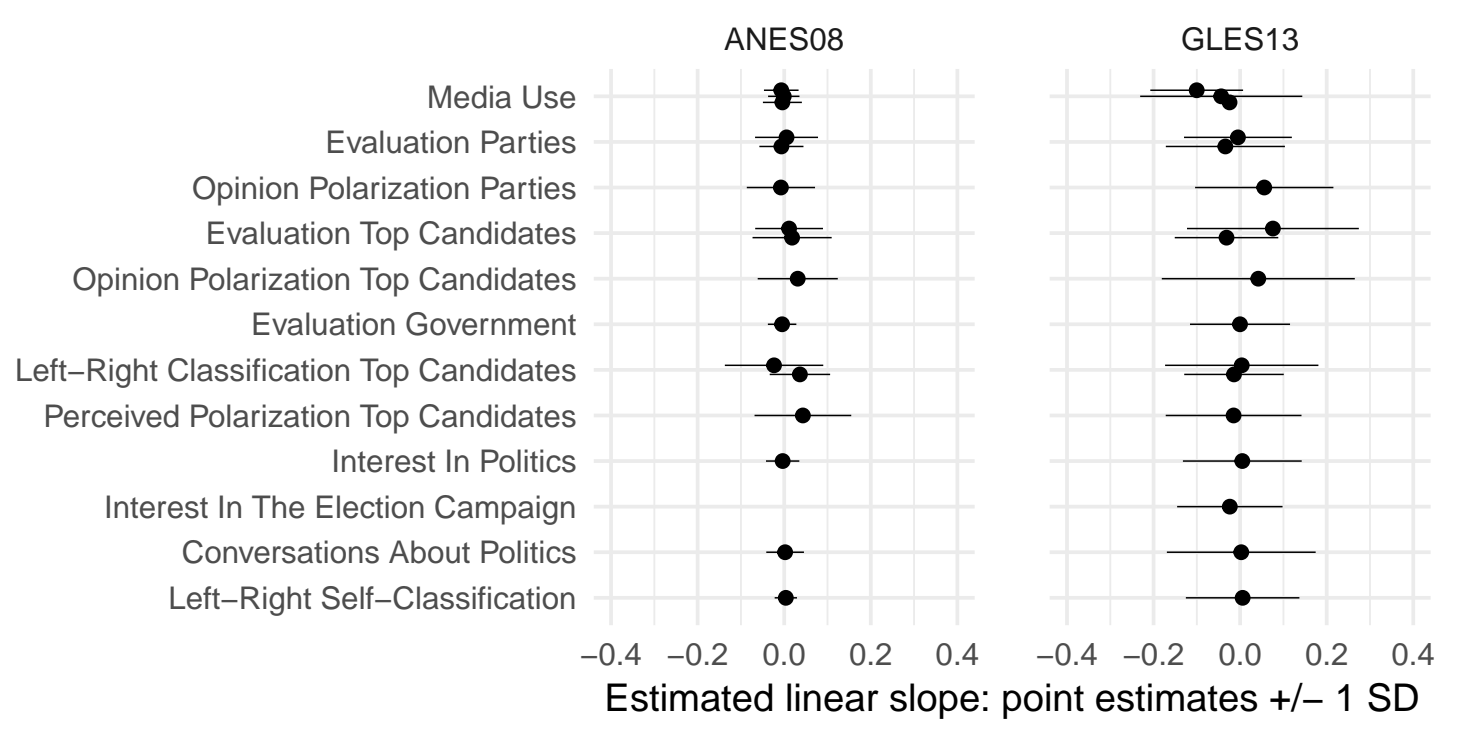

Figure 4. Growth curve estimates and their variability

Notes. The horizontal lines depict a range of $+/-1$ standard deviations of the individual slope estimates around the population point estimate. The individual slopes of approximately two thirds of the respondents fall in this range.

in the GLES 2013. Moreover, the relatively more varying slopes in perceived candidate positions are also related to the more pronounced measurement error in these variables (see Figure 2). If the observed variance is inflated per measurement occasion, it is likely that the variance in the observed changes will also be inflated. Considering the relative lack of inter-individual variation in intra-individual trajectories, most PLGCM involving the measures we investigated will therefore either lead to weakly correlated processes, or these correlated growth curves will most likely turn out to be parallel flat trajectories.

\section{Discussion}

Many typical election study measures (including news media use) turned out to be too stable to fulfill even the basic preconditions for establishing the RSM in typical panel designs and representative samples of the electorate. This applied to both studies and irrespective of whether we follow the analytical logic of the CLPM or the PLGCM. The results have relevant consequences for future research: They highlight the need for making the boundaries of dynamic spirals explicit in the RSM (see also Slater, 2015), and to consider all sources of variation when looking at possible reciprocal effects. Our first recommendation is that communication scholars pay more attention to measurement, e.g. by using more reliable measures (scales instead of single item measures), estimating the reliability of these measures whenever possible, and use statistical models that explicitly account for measurement error (Bachl \& Scharkow, 2018). In most cases, this will lead to higher stability estimates, and therefore less variance to explain, but it also makes it more likely to detect media or selection effects at all.

Concerning the fundamental questions of appropriate research designs to test the RSM, Slater (2007) notes that "[c]learly, the longitudinal design must fit the phenomenon 
under study" (p. 286; see also Slater, 2015). As we have demonstrated, the typical election campaign study may not be ideal to uncover reinforcing spirals, for several reasons: Media use, e.g. the number of days per week where one watches the news, does not vary much month-to-month. In order to uncover cumulative effects of media exposure, longer time lags (in the order of years rather than weeks or months) may be necessary. This takes us outside the realm of campaign effects into mid- to long-term cultivation or socialization processes (Kruikemeier \& Shehata, 2017). Alternatively, researchers could design studies with shorter time lags (e.g. daily or even more frequent measurements) to capture short-term effects that are more in line with previous experimental research (Otto, Thomas, \& Maier, 2018). While the amount of daily media exposure might no vary much, either, there will likely be more variation over time when researchers consider (actual or probable) exposure to specific content rather than general media use. Such linkage analyses (Scharkow \& Bachl, 2017) also match Slater's (2015) theoretical notion that it is exposure to media content, rather than general media use, which drives the spiraling process.

Another boundary condition for the RSM is that not only media use, but also the orientations, attitudes or behaviors of the respondents need to vary over time. As we have demonstrated, most political orientations and attitudes do not fulfill this condition, at least in the span of a typical election campaign. It might therefore be more promising to apply the RSM to conceptually less stable constructs (e.g., perceptions or emotions) rather than trait-like measures such as political interest or party preferences. In addition or alternatively, the RSM can be used to study populations other than typical voters, e.g. children and adolescents (Moeller \& de Vreese, 2015), or move to more volatile contexts, e.g. referendums (Schemer, 2012), or political systems. However, it can to be debated whether moving the research focus to presumably more dynamic environments is a useful endeavor when much political media effects research is explicitly or implicitly legitimized by the political importance of elections and election campaigns, which often display rather little dynamics.

Finally, we argue that it is necessary to explicitly match the theoretical formulation of the RSM to an appropriate statistical model. In the past, both the CLPM and the PLGCM have been applied to identify reinforcing spirals, with comparatively few justifications given for choosing one over the other (or even third options). However, since both models make strong assumptions about the causal processes under investigation, and often answer distinctly different questions, it seems prudent to explicitly state which parameters in the model supposedly match the spiral process, and which the reinforcing effects. Moreover, most of the original formulations of the RSM (Slater, 2007, 2015) suggest that the processes under investigation are clearly intra-individual rather than inter-individual. However, as pointed out by Baumgartner, van der Schuur, Lemmens, and te Poel (2017), the CLPMs

"do not disaggregate between-person differences from within-person changes over time. Therefore, common CLPM do not allow one to draw conclusions about individual change over time. If a cross-lagged path is found in a traditional CLPM, it is thus not clear whether this effect is due to longitudinal changes on the individual level, due to changes on the between-person level, or due to both types of effects" (p. 11).

The same argent is true for the PLGCM, since only the covariance of individual trajectories is modelled, which is a between-person estimate. Recently, Hamaker, Kuiper, 
and Grasman (2015) suggested a modified random intercept CLPM that accounts for both within- and between-person effects, with the cross-lagged effects on the within-person level. We agree with Baumgartner et al. (2017) that the RI-CLPM currently seems the best statistical model to empirically test the RSM, and their results seem to deliver on that promise in terms of model fit and interpretability. However, only few studies in communication research have used this model, so we have to wait whether this solves the statistical issues in identifying reinforcing spirals in communication research. At the very least, it will force researchers to think about and explicitly state what exactly these mutually reinforcing effects are and how they can be investigated empirically. 


\section{References}

Alwin, D. F. (2007). Margins of error: A study of reliability in survey measurement (Vol. 547). John Wiley \& Sons.

Bachl, M., \& Scharkow, M. (2018). Some suggestions on dealing with measurement error in linkage analyses. In C. Peter, T. Naab, \& R. Kühne (Eds.), Measuring media use and exposure: Recent developments and challenges. Herbert von Halem.

Bates, D., Maechler, M., Bolker, B., \& Walker, S. (2017). Lme4: Linear mixed-effects models using 'eigen' and s4. Retrieved from https://CRAN.R-project.org/package=lme4

Baumgartner, S. E., van der Schuur, W. A., Lemmens, J. S., \& te Poel, F. (2017). The relationship between media multitasking and attention problems in adolescents: Results of two longitudinal studies. Human Communication Research, 44 (1), 3-30.

Feingold, A. (2009). Effect sizes for growth-modeling analysis for controlled clinical trials in the same metric as for classical analysis. Psychological Methods, 14(1), 43-53. doi:10.1037/a0014699

Grimm, K. J., Ram, N., \& Estabrook, R. (2016). Growth modeling: Structural equation and multilevel modeling approaches. Guilford Publications.

Hamaker, E. L., Kuiper, R. M., \& Grasman, R. P. (2015). A critique of the cross-lagged panel model. Psychological Methods, 20(1), 102.

Heise, D. R. (1969). Separating reliability and stability in test-retest correlation. American Sociological Review, 34(1), 93-101. doi:10.2307/2092790

Kruikemeier, S., \& Shehata, A. (2017). News media use and political engagement among adolescents: An analysis of virtuous circles using panel data. Political Communication, $34(2), 221-242$.

Moeller, J., \& de Vreese, C. (2015). Spiral of political learning. Communication Research. doi:10.1177/0093650215605148

Otto, L., Thomas, F., \& Maier, M. (2018). Everyday dynamics of media skepticism and credibility. In K. Otto \& A. Köhler (Eds.), Trust in media and journalism (pp. 111-133). Springer.

R Core Team. (2016). R: A language and environment for statistical computing. Vienna, Austria: R Foundation for Statistical Computing. Retrieved from https://www. R-project.org/

Rattinger, H., Roßteutscher, S., Schmitt-Beck, R., Weßels, B., Wolf, C., Plischke, T., \& Wiegand, E. (2016). Short-term Campaign Panel 2013 (GLES). GESIS Data Archive. doi:10.4232/1.12561

Rosseel, Y. (2017). Lavaan: Latent variable analysis. Retrieved from https://CRAN. R-project.org/package=lavaan

Scharkow, M., \& Bachl, M. (2017). How measurement error in content analysis and selfreported media use leads to minimal media effect findings in linkage analyses: A simulation study. Political Communication, 34(3), 323-343.

Schemer, C. (2012). Reinforcing spirals of negative affects and selective attention to advertising in a political campaign. Communication Research, 39(3), 413-434. doi:10.1177/0093650211427141

Schemer, C., Matthes, J., \& Wirth, W. (2009). Applying latent growth models to the 
analysis of media effects. Journal of Media Psychology, 21(2), 85-89.

Slater, M. D. (2007). Reinforcing spirals: The mutual influence of media selectivity and media effects and their impact on individual behavior and social identity. Communication Theory, 17(3), 281-303. doi:10.1111/j.1468-2885.2007.00296.x

Slater, M. D. (2015). Reinforcing spirals model: Conceptualizing the relationship between media content exposure and the development and maintenance of attitudes. Media Psychology, 18(3), 370-395. doi:10.1080/15213269.2014.897236

Slater, M. D. (2017). Reinforcing spirals model. In P. Rössler, C. A. Hoffner, \& L. van Zoonen (Eds.), The International Encyclopedia of Media Effects. Hoboken: Wiley. Retrieved from http://dx.doi.org/10.1002/9781118783764.wbieme0118

Slater, M. D., \& Hayes, A. F. (2010). The influence of youth music television viewership on changes in cigarette use and association with smoking peers: A social identity, reinforcing spirals perspective. Communication Research, 37(6), 751-773. doi: $10.1177 / 0093650210375953$

The American National Election Studies. (2010). 2008-2009 Panel Study. Stanford University and the University of Michigan. Retrieved from www.electionstudies.org

Wickham, H. (2009). Ggplot2: Elegant graphics for data analysis. Springer-Verlag New York. Retrieved from http://ggplot2.org 